\title{
Impacto de los juegos reducidos sobre la toma de decisiones y la técnica en jugadores de fútbol sub-12: efecto del espacio cerrado
}

\section{Impact of small sided games on decision making and technique on U-12 football players: effect of closed area}

\author{
Salvador Pérez Muñoz ${ }^{1 *}$, David Recouvreur Encinas ${ }^{1}$, Antonio Sánchez Muñoz ${ }^{1}$, Alberto \\ Rodríguez Cayetano 1 \\ ${ }^{1}$ Universidad Pontificia de Salamanca, Salamanca, España. \\ * Correspondencia: Salvador Pérez Muñoz; sperezmu@upsa.es
}

\begin{abstract}
The aim of the research was to analyze how the practice of small sided games influences technique and decision-making in U-12 football players male, with modification of the space. There were $12 \mathrm{U}-$ 12 players participating. They carried out 20 training sessions based on small sided games of football on a playing field that is surrounded by walls generating an enclosed playing space. The sample showed normal values in the Saphiro-Wilk test. The main results show that there are significant differences in the pass and control variables, and there is a significant improvement in the execution and decision-making actions. Decision-making showed significant differences in all the indicators. Therefore, it improves the technique and decisions in specific game situations, through the use of small sided games, in this way it can be considered as a methodological resource to be used in these training phases.
\end{abstract}

\section{KEYWORDS}

Soccer learning; Small-sided games; Decision making; Technique; Tactics

\section{RESUMEN}

El objetivo de la investigación fue analizar cómo influyen la práctica de juegos reducidos sobre la técnica y la toma de decisiones en jugadores sub-12 de fútbol masculino, con modificación del espacio. Participaron 12 jugadores Sub-12. Realizaron 20 sesiones de entrenamiento basado en juegos reducidos de fútbol en un campo de juego que está rodeado con paredes generando un espacio 
de juego cerrado. La muestra presentó valores normales en la prueba de Saphiro-Wilk. Los principales resultados mostraron que existen diferencias significativas en las variables de pase y control, y se mejoran las acciones técnicas y la toma de decisiones de forma significativa. La toma de decisiones presentó diferencias significativas en todos los índices. Por lo tanto, mejora la técnica y las decisiones en situaciones específicas de juego, mediante el uso de los juegos reducidos, de esta forma se puede considera como un recurso metodológico a utilizar en estas etapas de formación.

\section{PALABRAS CLAVE}

Fútbol base; Juegos reducidos; Toma de decisiones; Técnica; Táctica

\section{INTRODUCCIÓN}

El fútbol es el deporte más famoso, más practicado y con mayor repercusión social (Joo, Hwang-Bo \& Jee, 2016), en la mayoría de los países del mundo. Las investigaciones sobre este deporte han evolucionado de forma notable en los últimos años, y aunque cada vez hay más estudios en jugadores jóvenes, todavía no son tan relevantes como en etapas superiores y de rendimiento (Clemente, Sarmento, Costa, Enes \& Lima, 2019a), necesitando más investigaciones que se centren en las etapas de formación deportiva (González-Villora, García-López \& Contreras-Jordán, 2015).

Dentro del campo del entrenamiento y la enseñanza en los deportes, la metodología se encuentra constante evolución con el objeto de proporcionar a los deportes colectivos una mayor especificidad (Pascual, Guillén \& Carbonell, 2017), huyendo de modelos tradicionales o clásicos que no tienen en cuenta el contexto de juego que separa los factores de rendimiento aislando el aprendizaje en compartimentos estanco donde el jugador es un simple ejecutor (Pascual et al., 2017; Pérez, Chamorro, Rodríguez, Sánchez \& De Mena, 2020; Práxedes, Del Villar, Pizarro \& Moreno 2018), poniendo el énfasis y el centro de atención en el profesor-entrenador (Teacher-Centered Approach) (González-Espinosa, Ibáñez \& Feu, 2017). Esta evolución lleva a otro modelo de entrenamiento y enseñanza innovador, novedoso o alternativo (Pérez et al., 2020) que interrelaciona todos los factores propios del deporte y del rendimiento, buscando una formación autónoma por parte del jugador (Castejón, 2015; González-Espinosa et al., 2017) más centrado en el alumno-jugador (Student-Centered Approach) (González-Espinosa et al., 2017), todo ello a través de tareas jugadas, juegos reducidos (JR) o small-sided games (SSGs), que reúnen este tipo de condiciones, convirtiéndose en uno de los recursos metodológicos de entrenamiento más populares (Clemente, Suárez-Arrones \& Gil, 2019c; Owen, Wong, Paul, \& Dellal, 2012). Este tipo de JRs se incluyen en el 
entrenamiento y la enseñanza de los deportes de forma novedosa (Pérez et al., 2019), porque tienen en cuenta la lógica interna, la especifidad y las condiciones que más se asemejan a la competición (Moran et al., 2019; Romero-Caballero \& Campos-Vázquez, 2020).

Los JRs sirven para mejorar el rendimiento de los jugadores, ya que permiten la adaptación de las reglas y del espacio al menor número de jugadores lo que va a permitir al entrenador reproducir las condiciones, las demandas y las incertidumbres propias de los partidos (Clemente et al., 2019b; Cristian et al., 2015; Owen, Twist \& Ford, 2004). Son de gran utilidad con independencia de la edad y el nivel de los jugadores, siendo especialmente adecuados en las etapas de formación (Casamichana, San Román, Calleja \& Castellano, 2015; Moran et al., 2019). Por lo tanto, reúnen la complejidad y especifidad del fútbol, donde el jugador puede mejorar todos los factores asociados al rendimiento (Dellal, Hill-Haas, Lago-Penas \& Chamari, 2011; Romero-Caballero \& CamposVázquez, 2020), y sobre todo, incrementar su participación en juego, lo que permitirá un mayor número de acciones y decisiones a tomar en condiciones contextuales de estrés y esfuerzo específico en el juego (Joo et al., 2016; Romero-Caballero \& Campos-Vázquez, 2020; Reche-Soto, Cardona, Díaz, Gómez-Carmona \& Pino-Ortega, 2019).

En este ámbito, es cierto que existen una buena cantidad de estudios científicos basados en la realización de JRs (Rampinini et al., 2007) como recurso metodológico de entrenamiento, desde estudios que analizan la cuantificación de la condición física y la carga interna (Dellal et al., 2011; Hill-Haas, Dawson, Impellizzeri \& Coutts, 2011; Moran et al., 2019; Owen et al., 2012), hasta los que se centran en aspectos técnicos-tácticos (Coutinho et al., 2020; Joo et al., 2016; Pascual et al, 2017; Reche-Soto et al., 2019; Sánchez et al., 2014; Serra-Olivares et al., 2019). Otros estudian el efecto de la manipulación de otras variables como: número de jugadores, espacio, demarcaciones, distribución de equipos, reglas y balón (Coutinho et al., 2019; Folgado, Bravo, Pereira \& Sampaio, 2019; González-Víllora et al., 2015; Lacome, Simpson, Cholley \& Buchheit, 2018; Moreira et al, 2016; Santos et al., 2020).

En el fútbol los JRs y la toma de decisiones, como elemento que se integra en la táctica, van íntimamente relacionadas, al ser uno de los aspectos más complejos e importantes en los deportes colectivos (Coutinho et al., 2019; Sánchez, Hernández-Mendo, Martínez, Reigal \& Chirosa, 2018). Por lo tanto, la toma de decisiones va a ser un elemento clave en las acciones del jugador, mejorando su creatividad para conseguir solucionar los problemas del fútbol, lo que le va a permitir una mejor comprensión del juego en todos los parámetros (Hill-Haas et al., 2011). Sin embargo, pese a la gran 
importancia que tiene la toma de decisiones no se trabaja lo suficiente en la enseñanza de los deportes (Sánchez et al., 2018).

En esta línea, considerando que no es un proceso lineal y normativo, hay estudios que analizan el conocimiento táctico y la toma de decisiones de jugadores de distinto nivel, (GonzálezVillora et al., 2015; Levi \& Jackson, 2018), con jugadores sub-12 que mejoran en el pase y el regate (Práxedes et al., 2018) y mejora de la toma de decisiones y comprensión del juego (González-Víllora et al., 2015; Mesquita, Farias \& Hastie, 2012; Farias, Ribeiro \& Hastie, 2015). Por último, SerraOlivares, González-Víllora y García-López (2011) concluyen que en las situaciones de 3x3 los jugadores toman mejores decisiones que en otro tipo de JRs.

Sin embargo, hay que manifestar que no existen estudios que lo hagan sobre la técnica y la toma de decisiones, como componente de la táctica, de los jugadores utilizando los JRs con variante de espacio rodeado de paredes, cerrado, lo que va a provocar que el juego tenga mayores niveles de continuidad, menos pérdidas de tiempo y que los estímulos sean relativamente más altos (Impellizzeri et al., 2006). Por todo ello el objetivo del estudio fue analizar cómo influyen la práctica de juegos reducidos de forma interrelacionado sobre la técnica y la toma de decisiones en jugadores sub-12 de fútbol, con modificación del espacio a través de metodología alternativa.

\section{MÉTODOS}

La investigación realizada es de tipo cuantitativa cuasiexperimental a través de un estudio de campo con un test, intervención y un re-test final, para comprobar el efecto de la intervención sobre la técnica, la táctica y la toma de decisiones. Pita y Pértegas (2002) indican que es una metodología de investigación cuantitativa, ya que se recogen datos observables, medibles, cuantificables y se ofrecen unos resultados numéricos. Además, se utilizó la metodología observacional para la recogida de datos.

\subsection{Participantes}

La muestra la forman 12 jugadoras alevines en un solo grupo de estudios, seis de ellos fueron seleccionados con la Selección de Castilla y León Sub-12 para la disputa del Campeonato de España de su categoría, por lo tanto, se pueden considerar como jugadores de alto nivel de pericia, al diferir su nivel de rendimiento a otros jugadores con edad y nivel competitivo similar (Serra-Olivares, García-López, Calderón \& Cuevas-Campos, 2015), con edades comprendidas entre los 11-12 años $(\mathrm{M}=11.42 ; \pm .515)$. De los cuales dos son porteros (que juegan como jugadores de campo, es decir 
Pérez Muñoz et al.

que al analizar los datos se nombran la posición de portero habitual en los partidos, pero han intervenido como jugadores de campo), tres son defensas, cuatro medios y tres delanteros.

\subsection{Material}

Toda la parte experimental se desarrolló en las mismas instalaciones del club donde entrenan los jugadores de forma habitual, tanto campo, como material y vestuarios. Las mediciones se realizaron por el mismo evaluador, que había sido formado previamente a la toma de datos en varias tandas de test.

El test realizado fue el siguiente:

- Se realizó un 3x3 en un terreno de juego de 20x10 m., durante cinco minutos, cuya principal característica es que estará cerrado, de manera que el balón no saldría salvo en aquellas situaciones en las que sobrepase la altura (1.26 m.).

○ El juego será libre respetando las reglas de fútbol-7, no existirá fuera de juego y los saques de banda se harán con el pie. El saque de centro se realizará mediante un bote neutral. Si el balón sale, el juego lo reanudará el equipo contrario, desde su propia portería. El objetivo final es conseguir el mayor número de goles. En el fondo de cada portería, se colocarán dos balones más para iniciar el juego de manera inmediata. No hay portero y ningún jugador podrá asumir dicho rol. Si uno toca el balón con la mano será penalti sin oposición.

- Para determinar los índices de ejecución-técnica y de toma de decisiones-táctica, se utilizó la fórmula validada por Vera, Pino, Romero \& Moreno (2007) que mide la actuación de los jugadores en situaciones de juego reducido. Se midieron los índices de: ejecución-técnica, decisional (táctica) y técnico-táctico. La fórmula utilizada fue:

Índice de Actuación $=$ Aciertos $/($ Aciertos + Errores $)$

○ Este índice oscila entre cero y uno, donde uno se corresponde al mayor índice de acierto.

- Para medir la toma de decisiones y las ejecuciones en situación real de juego se ha utilizado como base el instrumento propuesto para baloncesto por French y Thomas, principalmente, donde se separa el componente cognitivo-decisional del rendimiento de la ejecución de las habilidades motrices específicas (French \& Thomas, 1987; Nevett, Rovegno, Babiarz \& McCaughtry, 2001).

- Se miden las siguientes variables con una ficha creada ah-hoc, desde una triple perspectiva, en primer lugar, la ejecución de la acción (técnica), en segundo lugar, la toma de decisiones (táctica) y por último de forma conjunta técnica-táctica de cada una de las 
acciones, en ambos casos analizando la acción si lo realizaban de forma correcta o incorrecta.

- Las variables analizadas fueron: Pase; Control; Disparo (tiro); Regate; Conducción; Acoso; Desmarque; Robo; Marcaje; Apoyo; Pase con muro; Entrada; Vigilancia; Cobertura; Interceptación; Finta; Pared con muro; Temporización; Anticipación; Pared con compañero y Carga.

- A continuación, se indica la codificación de las variables utilizada durante la observación de la ejecución y toma de decisiones utilizada (tabla 1):

Tabla 1. Codificación de acciones técnicas y tácticas.

\begin{tabular}{|c|c|c|}
\hline Criterio & Categoría & Descripción \\
\hline \multirow{4}{*}{ Pase } & EJCO & $\begin{array}{l}\text { El pase llega bien dirigido al compañero, con la fuerza suficiente, y } \\
\text { realizado con la superficie de contacto correcta. }\end{array}$ \\
\hline & EJINCO & $\begin{array}{l}\text { El pase no llega bien dirigido al compañero, la fuerza es excesiva, y no } \\
\text { se realiza con la superficie de contacto adecuada }\end{array}$ \\
\hline & DECO & $\begin{array}{l}\text { El jugador realiza un pase desde una distancia cualquiera como mejor } \\
\text { opción para mantener la posesión del balón. }\end{array}$ \\
\hline & DEINCO & $\begin{array}{l}\text { El jugador realiza un pase de forma inapropiada o cuando tiene } \\
\text { mejores opciones o posición para el lanzamiento. }\end{array}$ \\
\hline \multirow{4}{*}{ Control } & EJCO & $\begin{array}{l}\text { Busca el balón en el lugar esperado, interviene con la parte del cuerpo } \\
\text { más segura y se orienta preparándose para la acción posterior. }\end{array}$ \\
\hline & EJINCO & $\begin{array}{l}\text { No busca el balón y lo esperado, no interviene con la parte del cuerpo } \\
\text { más segura y no se orienta preparándose para la acción posterior }\end{array}$ \\
\hline & DECO & $\begin{array}{l}\text { El jugador es el más apropiado para dominar el balón y dejarlo en } \\
\text { disposición de jugarlo en la acción posterior. }\end{array}$ \\
\hline & DEINCO & $\begin{array}{l}\text { No es el jugador más apropiado para dominarlo y dejarlo en } \\
\text { disposición de jugarlo en la acción posterior. }\end{array}$ \\
\hline \multirow{3}{*}{ Disparo } & EJCO & $\begin{array}{l}\text { El disparo llega bien dirigido a la portería, con la fuerza suficiente, y } \\
\text { realizado con la superficie de contacto correcta. }\end{array}$ \\
\hline & EJINCO & $\begin{array}{l}\text { El disparo no llega bien dirigido a la portería, con la fuerza } \\
\text { insuficiente, y es realizado con la superficie de contacto incorrecta. }\end{array}$ \\
\hline & DECO & El jugador realiza un disparo desde una distancia cualquiera como \\
\hline
\end{tabular}


mejor opción para finalizar el ataque.

DEINCO El jugador realiza un disparo desde una distancia cualquiera de forma inapropiada ya tiene mejores opciones de disparo otro compañero.

EJCO Logra avanzar con el balón desbordando al adversario

EJINCO No logra avanzar con el balón. No logra desbordar al adversario

Regate

DECO No hay opción de pase. Sorprende al adversario. Es la mejor opción para conservar y/o progresar con el balón.

DEINCO Hay opción de realizar un pase para progresar. No es la mejor opción para conservar y/o progresar con el balón.

EJCO El jugador conduce la pelota interponiendo su cuerpo entre ella y el rival, y llevándolo cerca del pie.

EJINCO El jugador conduce la pelota sin interponer su cuerpo entre ella y el rival, y llevándola lejos del pie.

Conducción

DECO El jugador conduce porque tiene una ocasión clara de marcar, o le han dejado espacio para avanzar sin un compañero mejor situado a quien pasar

DEINCO El jugador no conduce el hacía la línea de fondo cuando tiene una ocasión clara de marcar, o lo hace teniendo un compañero mejor situado a quien pasar

EJCO El jugador se acerca al rival con balón con actitud defensiva.

EJINCO El jugador se acerca al rival con balón sin actitud defensiva.

Acoso

DECO El jugador acosa al rival porque tiene la ocasión de evitar el avance con el balón por parte del jugador rival con intensidad defensiva.

DEINCO El jugador no acosa al rival, aunque tenga ocasión de evitar el avance con el balón, espera al rival y no muestra intensidad defensiva.

EJCO Se escapa del jugador que realiza el marcaje, con movimiento de separación del rival.

EJINCO No se escapa del jugador que realiza el marcaje, sin movimiento de Desmarque separación del rival.

DECO Ayuda a avanzar con su acción la progresión del propio equipo escapando de la acción del rival.

DEINCO No ayuda a avanzar con su acción la progresión del propio equipo 
escapando de la acción del rival.

EJCO El jugador roba el balón interponiendo su cuerpo entre el y el rival, y no hace falta, al contrario.

EJINCO El jugador no roba el balón interponiendo su cuerpo entre el y el rival y Robo golpea en falta, al contrario.

DECO El jugador roba el balón porque el rival le deja espacio entre él y el balón y es la mejor opción para recuperar el balón.

DEINCO El jugador no roba el balón porque el rival no deja espacio entre él y el balón y no es la mejor opción para recuperar el balón.

EJCO Marca al jugador que tiene asignado por el espacio de juego.

Marcaje

EJINCO No marca al jugador que tiene asignado por el espacio de juego.

DECO Ayuda a frenar la progresión del equipo rival.

DEINCO No ayuda a frenar la progresión del equipo rival.

EJCO El jugador atacante sin balón ofrece una buena oportunidad de pase al compañero con balón, liberándose del marcaje del

EJINCO El jugador atacante sin balón no ofrece una buena oportunidad de pase

Apoyo

DECO Ayuda a mantener la posesión y avanzar con su acción en el ataque de su equipo.

DEINCO No realiza una ayuda para mantener la posesión y avanzar con su acción en el ataque de su equipo.

EJCO El pase llega bien dirigido al compañero, con la fuerza suficiente, y realizado con la superficie de contacto correcta utilizando el muro.

EJINCO El pase no llega bien dirigido al muro, la fuerza es excesiva, y no se realiza con la superficie de contacto adecuada utilizando el muro.

Pase con muro

DECO El jugador realiza un pase desde una distancia cualquiera como mejor opción para mantener la posesión utilizando el muro.

DEINCO El jugador realiza un pase de forma inapropiada al muro o cuando tiene mejores opciones de pase o posición para el lanzamiento.

EJCO Ejecuta la acción al jugador con balón con la fuerza suficiente, y

Entrada realizado con la superficie de contacto correcta.

EJINCO Ejecuta la acción al jugador con balón con la fuerza suficiente, y 
realizado con la superficie de contacto correcta.

DECO El jugador realiza la acción como mejor opción para recupera la posesión del balón tirándose al suelo.

DEINCO El jugador realiza la acción cuando tenía otra opción para recupera la posesión del balón y no se tira al suelo.

\begin{tabular}{|c|c|c|}
\hline \multirow{5}{*}{ Vigilancia } & EJCO & $\begin{array}{l}\text { Realiza un marcaje individual al jugador del equipo rival cuando su } \\
\text { equipo esta atacando. }\end{array}$ \\
\hline & EJINCO & $\begin{array}{l}\text { No realiza un marcaje individual al jugador del equipo rival cuando su } \\
\text { equipo esta atacando. }\end{array}$ \\
\hline & DECO & El defensor más alejado del ataque se no interviene en la fase de ataque \\
\hline & & $\begin{array}{l}\text { y se fija en el rival por si recibe la pelota ante una posible pérdida de } \\
\text { balón de su equipo. }\end{array}$ \\
\hline & DEINCO & $\begin{array}{l}\text { El defensor más alejado del ataque interviene en la fase de ataque y no } \\
\text { fija en el rival por si recibe la pelota ante una posible pérdida de balón } \\
\text { de su equipo. }\end{array}$ \\
\hline \multirow{5}{*}{ Cobertura } & EJCO & El jugador se sitúa detrás de un compañero que defiende al poseedor de \\
\hline & EJINCO & $\begin{array}{l}\text { balón, para entrar en acción en caso de que este sea desbordado. } \\
\text { El jugador no se sitúa en posición de ayuda a un compañero que } \\
\text { defiende al atacante con balón. }\end{array}$ \\
\hline & $\mathrm{DECO}$ & $\begin{array}{l}\text { El jugador deja al rival propio para colocarse en posición de ayuda a su } \\
\text { compañero que defiende al atacante con balón. }\end{array}$ \\
\hline & DEINCO & El jugador no deja al rival propio para colocarse en posición de ayuda a \\
\hline & & su compañero que defiende al atacante con balón. \\
\hline \multirow{4}{*}{ Interceptación } & EJCO & $\begin{array}{l}\text { Ejecuta la acción al jugador que va a recibir el balón con la fuerza } \\
\text { suficiente y la superficie de contacto necesaria para obtener el balón. }\end{array}$ \\
\hline & EJINCO & $\begin{array}{l}\text { Ejecuta la acción al jugador que va a recibir el balón con fuerza } \\
\text { insuficiente y la superficie de contacto incorrecta para obtener el balón. }\end{array}$ \\
\hline & DECO & $\begin{array}{l}\text { El jugador realiza la acción como mejor opción para recupera el balón } \\
\text { antes de que lo reciba el rival, sin provocar infracción y obteniendo la } \\
\text { posesión del balón. }\end{array}$ \\
\hline & DEINCO & $\begin{array}{l}\text { El jugador no realiza la acción como mejor opción para recupera el } \\
\text { balón antes de que lo reciba el rival, provoca una infracción y no }\end{array}$ \\
\hline
\end{tabular}




\begin{tabular}{|c|c|c|}
\hline & & lel balón. \\
\hline \multirow{4}{*}{ Finta } & EJCO & $\begin{array}{l}\text { Logra engañar al adversario desbordándolo con su cuerpo y con } \\
\text { velocidad adecuada. }\end{array}$ \\
\hline & EJINCO & $\begin{array}{l}\text { No logra engañar al adversario con su cuerpo, desbordándolo con } \\
\text { velocidad adecuada. }\end{array}$ \\
\hline & $\mathrm{DECO}$ & $\begin{array}{l}\text { Sorprende al adversario con su movimiento rápido y es la mejor opción } \\
\text { ayudar al compañero con balón. }\end{array}$ \\
\hline & DEINCO & $\begin{array}{l}\text { No logra sorprende al adversario con su movimiento rápido y no es la } \\
\text { mejor opción ayudar al compañero con balón. }\end{array}$ \\
\hline \multirow{4}{*}{$\begin{array}{l}\text { Pared con } \\
\text { muro }\end{array}$} & EJCO & $\begin{array}{l}\text { Realiza una entrega y devolución del balón a un toque utilizando el } \\
\text { muro. }\end{array}$ \\
\hline & EJINCO & $\begin{array}{l}\text { La pared no llega bien dirigido al muro, la fuerza es excesiva, y no se } \\
\text { realiza con la superficie de contacto adecuada utilizando el muro. }\end{array}$ \\
\hline & DECO & $\begin{array}{l}\text { La acción sirve para progresar en el juego. Se supera la posición de al } \\
\text { menos un adversario. }\end{array}$ \\
\hline & DEINCO & $\begin{array}{l}\text { La acción no sirve para progresar en el juego y no se supera a ningún } \\
\text { adversario. }\end{array}$ \\
\hline \multirow{4}{*}{ Temporización } & EJCO & $\begin{array}{l}\text { El jugador se sitúa en la línea de ataque del poseedor del balón, } \\
\text { ralentizando la acción ofensiva y evita ser desbordado. }\end{array}$ \\
\hline & EJINCO & $\begin{array}{l}\text { El jugador no se sitúa en la línea de ataque del poseedor del balón, no } \\
\text { ralentiza la acción ofensiva y permite ser desbordado. }\end{array}$ \\
\hline & $\mathrm{DECO}$ & $\begin{array}{l}\text { La acción sirve para ralentiza y evitar la progresión en el juego del } \\
\text { rival y no permite ser superado en su posición. }\end{array}$ \\
\hline & DEINCO & $\begin{array}{l}\text { La acción no sirve para ralentiza y evitar la progresión en el juego del } \\
\text { rival, y permite ser superado en su posición. }\end{array}$ \\
\hline \multirow{3}{*}{ Anticipación } & EJCO & $\begin{array}{l}\text { Ejecuta la acción al jugador que va a recibir el balón con la fuerza } \\
\text { suficiente y la superficie de contacto necesaria para evitar que el rival } \\
\text { obtenga la posesión del balón. }\end{array}$ \\
\hline & EJINCO & $\begin{array}{l}\text { Ejecuta la acción al jugador que va a recibir el balón con la fuerza } \\
\text { insuficiente y la superficie de contacto incorrecta para evitar que el } \\
\text { rival mantenga la posesión del balón. }\end{array}$ \\
\hline & $\mathrm{DECO}$ & El jugador realiza la acción como mejor opción para recupera el balón \\
\hline
\end{tabular}


antes de que lo reciba el rival, sin provocar infracción y evitando que el rival mantenga la posesión del balón.

DEINCO El jugador no realiza la acción como mejor opción para recupera el balón antes de que lo reciba el rival, provoca una infracción y no evita que el rival mantenga la posesión del balón.

\begin{tabular}{|c|c|c|}
\hline \multirow{4}{*}{$\begin{array}{l}\text { Pared con } \\
\text { compañero }\end{array}$} & EJCO & $\begin{array}{l}\text { Realiza una entrega y devolución del balón a un toque entre dos } \\
\text { jugadores. }\end{array}$ \\
\hline & EJINCO & No se realiza la devolución del balón mediante un solo toque. \\
\hline & DECO & $\begin{array}{l}\text { La acción sirve para progresar en el juego. Se supera la posición de al } \\
\text { menos un adversario. }\end{array}$ \\
\hline & DEINCO & $\begin{array}{l}\text { La acción no sirve para progresar en el juego y no se supera a ningún } \\
\text { adversario. }\end{array}$ \\
\hline \multirow{4}{*}{ Carga } & EJCO & $\begin{array}{l}\text { Ejecuta la acción al jugador con balón con la fuerza suficiente, y } \\
\text { realizado con la superficie de contacto correcta. }\end{array}$ \\
\hline & EJINCO & $\begin{array}{l}\text { Ejecuta la acción al jugador con balón con la fuerza suficiente, y } \\
\text { realizado con la superficie de contacto correcta. }\end{array}$ \\
\hline & DECO & $\begin{array}{l}\text { El jugador realiza la acción como mejor opción para recupera la } \\
\text { posesión del balón sin tirarse al suelo. }\end{array}$ \\
\hline & DEINCO & $\begin{array}{l}\text { El jugador realiza la acción cuando tenía otra opción para recupera la } \\
\text { posesión del balón y se tira al suelo. }\end{array}$ \\
\hline
\end{tabular}

*EJOC: Ejecución correcta; EJINCO: Ejecución Incorrecta; DECO: Decisión Correcta; DEINCO: Decisión Incorrecta.

\subsection{Procedimiento}

Para la realización del estudio se contactó con todos los responsables del club, tanto directos, como coordinadores y técnicos. A continuación, se solicitó consentimiento informado a los padres y madres para participar en el estudio y una vez obtenido y firmado se comenzó con el estudio. En este sentido se le permitía faltar un día al entrenamiento, durante todo el proceso. Se llevó a cabo siguiendo escrupulosamente las normas deontológicas reconocidas en la Declaración de Helsinki 2013. Convenio de Oviedo y siguiendo las recomendaciones de Buena Práctica Clínica de la CEE (documento 111/3976/88 de julio de 1990) y la normativa legal vigente española que regula la investigación clínica en humano (Real Decreto 561/1993 sobre ensayos clínicos).

El espacio utilizado es específico al material disponible, ya que es el único club que tiene un espacio cerrado con las dimensiones de 20x10 m., (33.3 $\mathrm{m}^{2}$ por jugador). Se realizaron un total de 20 
sesiones, más dos sesiones de toma de datos tanto test como re-test basada en JRs en espacio cerrado con metodología moderna y siempre con direccionalidad en el ataque y la defensa, con una duración de $40 \mathrm{~m}$. de intervención por sesión específica, con una duración de 90 minutos por entrenamiento, además de jugar un partido de competición a la semana.

Los objetivos y contenidos de las sesiones realizadas se distribuyen de la siguiente forma: todas tienen componente específico: ataque-defensa, compañeros-adversarios y técnica - táctica, tanto en situaciones de igualdad (16 sesiones), como en superioridad e inferioridad (cuatro sesiones) en espacio limitado por paredes. Se trabajan las variables técnico-tácticas específicas del estudio con un objetivo principal de cada sesión, realizado de la siguiente forma:

- Sesión 1: Pase; Sesión 2: Control; Sesión 3: Disparo (tiro); Sesión 4: Pared con compañero; Sesión 5: Desmarque; Sesión 6: Pase y control; Sesión 7: Conducción; Sesión 8: Acoso; Sesión 9: Marcaje; Sesión 10: Robo; Sesión 11: Anticipación; Sesión 12: Apoyo; Sesión 13: Pase con muro; Sesión 14: Entrada y carga; Sesión 15: Vigilancia; Sesión 16: Cobertura; Sesión 17: Interceptación; Sesión 18: Finta y Regate; Sesión 19: Pared con muro; Sesión 20: Temporización.

- Las sesiones en las que se trabaja en superioridad - inferioridad numérica fueron: $\mathrm{n}^{\mathrm{o}} 10^{\mathrm{a}}, 13^{\mathrm{a}}$, $18^{\mathrm{a}}$ y $20^{\mathrm{a}}$. El resto de las sesiones se han trabajado en situación de igualdad numérica en 3x3.

La ficha de observación creada ad-hoc fue evaluada y revisada por cuatro expertos del área, con dos doctores en Ciencias del Deporte, uno en Ciencias de la Educación y un Entrenador Nacional de fútbol para comprobar y evaluar la tabla de observación, llegando entre todos al formato final de ficha utilizada. Previamente a la realización de la intervención, para evitar errores, se les permitió a los jugadores que practicasen la prueba en dos sesiones anteriores al inicio de la investigación en las mismas condiciones que las realizadas en el test. Esto, además, permitió el entrenamiento del evaluador principal del estudio, así como la utilización como ensayo del instrumento de evaluación final utilizado.

\subsection{Análisis estadístico}

Para el tratamiento estadístico de los datos se utilizó el programa estadístico SPSS Statistics 20.0. Se calcularon los descriptivos (media y desviación típica). Se comprobó la normalidad de la muestra por medio de la Saphiro-Wilk. Se analizaron los efectos del programa de entrenamiento comparando los datos obtenidos en el test y el re-test a través de la prueba $t$ Student para muestras relacionadas, así como el análisis univariante ANOVA para comprobar si existen diferencias 
significativas en función del puesto de juego. Las diferencias entre los resultados se consideran significativas si $p \leq .05$.

\section{RESULTADOS}

Tras la intervención realizada, los jugadores presentan mejores valores (tabla 2), en el total de acciones en el re-test con respecto al test, 440 y 441 respectivamente. Destaca el mayor número de acciones en los elementos técnicos de pase, control y disparo, con respecto al test inicial. Por el contrario, el resto de las acciones son menores en el re-test con respecto al test, especialmente provocados estos resultados por la mejora del pase y del control. De forma general, existen diferencias significativas $(p<.05)$ en las variables: pase y control, mejorando el número de acciones tras la intervención, mientras que también existen en las variables: regate, acoso, desmarque, robo, marcaje, pase con muro, entrada, vigilancia y cobertura, sin embargo, en este caso en el retest los resultados son menores.

Tabla 2. Descriptivos y diferencias significativas: variables técnico-tácticas test y retest.

\begin{tabular}{llllll}
\hline & Test & \multicolumn{3}{l}{ Re-test } & Sig. \\
\hline & Total & & Media (DT) & Media & \\
& 107 & $8.92(.289)$ & 149 & $12.42(.515)$ & $.000^{*}$ \\
\hline Pase & 49 & $4.08(.289)$ & 83 & $6.92(.289)$ & $.000^{*}$ \\
Disparo & 37 & $3.08(.289)$ & 41 & $3.42(.515)$ & .104 \\
Regate & 32 & $2.67(.492)$ & 26 & $2.17(.389)$ & $.026^{*}$ \\
Conducción & 28 & $2.33(.492)$ & 23 & $1.92(.515)$ & .096 \\
Acoso & 25 & $2.08(.289)$ & 18 & $1.5(.522)$ & $.002^{*}$ \\
Desmarque & 22 & $1.83(.389)$ & 16 & $1.33(.492)$ & $.026^{*}$ \\
Robo & 21 & $1.75(.452)$ & 13 & $1.08(.289)$ & $.005^{*}$ \\
Marcaje & $1.67(.492)$ & 13 & $1.08(.289)$ & $.002^{*}$ \\
Apoyo & $1.25(.452)$ & 9 & $0.75(.452)$ & .082 \\
Pase con muro & 15 & $1(.00)$ & 7 & $.58(.515)$ & $.017^{*}$ \\
Entrada & 12 & $1(.00)$ & 6 & $.5(.5)$ & $.007^{*}$ \\
Vigilancia & 12 & $.92(.289)$ & 5 & $.42(.515)$ & $.007^{*}$ \\
Cobertura & 11 & $.92(.289)$ & 5 & $.42(.515)$ & $.026^{*}$ \\
Interceptación & 11 & $.83(.389)$ & 5 & $.42(.515)$ & .096 \\
\hline
\end{tabular}




\begin{tabular}{llllll}
\hline Finta & 6 & $.5(.522)$ & 5 & $.42(.515)$ & .777 \\
Pared con muro & 6 & $.5(.522)$ & 5 & $.42(.515)$ & .339 \\
Temporización & 5 & $.42(.515)$ & 4 & $.33(.492)$ & .754 \\
Anticipación & 5 & $.33(.492)$ & 4 & $.28(.453)$ & .339 \\
Pared con compañero & 3 & $.25(.452)$ & 2 & $.17(.189)$ & .674 \\
Carga & 3 & $.25(.452)$ & 2 & $.17(.189)$ & .339 \\
Total & 440 & & 441 & & \\
$* *_{p \leq .05}$ & & & &
\end{tabular}

De forma general las acciones técnicas realizadas de forma correcta (ATEC) mejoran tras la intervención, aunque no presentando diferencias significativas. En el caso de las acciones técnicas ejecutadas de forma incorrecta (ATEIC) la media baja tras la intervención, presentando diferencias significativas. En lo que respecta a la toma de decisiones correctas o acciones tácticas (ATAC) se mejora la media y muestra diferencias significativas. Por último, en las acciones tácticas incorrectas (ATAIC) bajan y muestran diferencias significativas (tabla 3).

Tabla 3. Descriptivos y diferencias significativas variables técnico-tácticas: Correctas e incorrectas.

\begin{tabular}{lcccc}
\hline & Media & DT & Suma & Sig. \\
\hline Acciones técnicas correctas test (ATECT) & 26.17 & 7.371 & 314 & \multirow{2}{*}{.129} \\
Acciones técnicas correctas retest (ATECRT) & 30.25 & 9.275 & 363 & \\
Acciones técnicas incorrectas test (ATEIT) & 10.50 & 2.747 & 126 & $.000^{*}$ \\
Acciones técnicas incorrectas retest (ATEIRT) & 6.50 & 1.784 & 78 & \\
\hline Acciones tácticas correctas test (ATACT) & 26.50 & 6.113 & 318 & \\
Acciones tácticas correctas retest (ATACRT) & 31.42 & 9.130 & 377 & $.034^{*}$ \\
Acciones tácticas incorrectas test (ATAIT) & 10.17 & 3.460 & 122 & $.000^{*}$ \\
Acciones tácticas incorrectas retest (ATAIRT) & 5.33 & 0.985 & 64 & \\
\hline
\end{tabular}

$* p \leq .05$

Por puesto de juego, los principales resultados en el test (tabla 4) muestran que los que juegan de porteros, habitualmente, son los que menos acciones de ejecución técnica correcta realizan, seguida por el resto de puesto. Tras la intervención, son los defensas los que presentan mayores mejoras, seguida por medios, porteros y delanteros. En cuanto a la media de acciones incorrectas, en 
este caso son los porteros los que presentan una media más baja, seguido por defensas, medios y delanteros, respectivamente. No existiendo diferencias significativas por puesto de juego.

En las acciones tácticas en el test, de nuevo, son los porteros los que obtienen menor media de decisiones correctas, seguida por los delanteros, medios y defensas. Por el contrario, en el restest son los defensas y medios lo que presentan mejor media de toma de decisiones correcta, respectivamente, seguido por los delanteros y en último lugar están los porteros en las acciones correctas (tabla 4). Las decisiones incorrectas en el test son delanteros los que presentan mayor media de errores, seguido por los medios, defensas y en último lugar los porteros, de acciones tácticas incorrectas. En el caso del retest, en las acciones tácticas incorrectas son los defensas los que obtienen mejores resultados de media, seguido por los porteros, medios y delanteros. Los jugadores que juegan de medios muestran diferencias significativas $(p<.05)$ en la reducción de los errores tras la intervención, mientras que el resto no muestran diferencias significativas. Por último, en la ANOVA no existen diferencias significativas entre el puesto de juego y las variables técnico-tácticas analizadas (tabla 5).

Tabla 4. Variables técnico-tácticas: Media y diferencias significativas entre cada puesto: habitual de juego.

\begin{tabular}{|c|c|c|c|c|c|c|c|c|}
\hline & \multicolumn{2}{|c|}{ Portero } & \multicolumn{2}{|l|}{ Defensa } & \multicolumn{2}{|c|}{ Medio } & \multicolumn{2}{|c|}{ Delantero } \\
\hline & Media & Sig. & Media & Sig. & Media & Sig. & Media & Sig. \\
\hline ATECT - ATECRT & $18-21$ & .205 & $27.67-33.67$ & .625 & $28.0-32.25$ & .276 & $27.67-30.33$ & .208 \\
\hline ATEIT - ATEIRT & $9.5-7.5$ & .295 & $10.33-5.67$ & .118 & $10.75-7.0$ & $.036 *$ & $11.0-6.0$ & .57 \\
\hline ATACT - ATACRT & $21-23.5$ & .126 & $28.67-34.67$ & .554 & $28.0-34.0$ & .130 & $26.0-30.0$ & .115 \\
\hline ATAIT - ATAIRT & $6.5-5$ & .50 & $9.33-4.67$ & .256 & $10.75-5.25$ & $.025 *$ & $12.67-6.33$ & .11 \\
\hline
\end{tabular}

Tabla 5. Variables técnico-tácticas descriptivos y ANOVA: test y retest por puesto habitual de juego.

\begin{tabular}{lccccc}
\hline & Portero & Defensa & Medio & Delantero & Sig. \\
\hline ATECT & $18(2.82)$ & $27.67(5.69)$ & $28(10.0)$ & $27.67(5.68)$ & .450 \\
ATECRT & $21(1.41)$ & $33.67(14.84)$ & $32.25(8.42)$ & $30.33(5.86)$ & .519 \\
ATEIT & $9.5(.707)$ & $10.33(3.22)$ & $10.75(3.86)$ & $11(2.65)$ & .956 \\
ATEIRT & $7.5(.707)$ & $5.67(.577)$ & $7.0(2.16)$ & $6(2.46)$ & .672 \\
\hline
\end{tabular}




\begin{tabular}{|c|c|c|c|c|c|}
\hline ATACT & $21(1.45)$ & $28.67(.577)$ & $28(9.13)$ & $26(6.08)$ & .584 \\
\hline ATACRT & $23.5(.71)$ & 34.67 (1.419) & $34(8.68)$ & $30(7.0)$ & .580 \\
\hline ATAIT & $6.5(2.12)$ & $9.33(4.73)$ & $10.75(2.63)$ & $12.67(2.51)$ & .268 \\
\hline ATAIRT & $5(.0)$ & $4.67(.577)$ & $5.25(.50)$ & $6.33(1.53)$ & .191 \\
\hline
\end{tabular}

En lo que se refiere a los índices de actuación, los resultados muestran que todos los índices mejoran tras la intervención, tanto el de ejecución-técnica, como el de toma de decisiones-táctica, y, cómo no, el índice técnico-táctico total, existiendo diferencias significativas entre los índices de actuación tras la intervención (tabla 6).

Tabla 6. Resultados medios de los índices de actuación técnico-tácticas test y retest.

\begin{tabular}{lccc}
\hline & Media & DT & Sig. \\
\hline Índice de Ejecución-Técnica test (IETET) & .71 & .061 & $.000 *$ \\
Índice de Ejecución-Técnica retest (IETERT) & .82 & .059 & \\
\hline Índice de Actuación Táctica test (IATACT) & .72 & .056 & $.000^{*}$ \\
Índice de Actuación Táctica retest (IATACRT) & .85 & .035 & .041 \\
Índice de Actuación Técnico-Táctica test (IATTT) & .72 & $.000 *$ \\
Índice de Actuación Técnico-Táctica retest (IATTRT) & .83 & .041 & \\
\hline$* p \leq 05$ & & &
\end{tabular}

Por último, en relación con el puesto de juego los resultados muestran que todos los puestos mejoran tras la intervención en todos los índices analizados técnico, táctico y técnico-táctico. Los defensas muestran diferencias significativas $(p<.05)$ en el índice conjunto técnico-táctico, los medios y delanteros en todos los índices analizados, por lo tanto, son los que muestran mayores mejoras en la intervención (tabla 7). Si bien de forma conjunta, en la ANOVA no existen diferencias significativas entre el puesto de juego y los índices analizados (tabla 8). 
Tabla 7. Índices de actuación: Media y diferencias significativas: por puesto habitual de juego

\begin{tabular}{|c|c|c|c|c|c|c|c|c|}
\hline & \multicolumn{2}{|c|}{ Portero } & \multicolumn{2}{|c|}{ Defensa } & \multicolumn{2}{|c|}{ Medio } & \multicolumn{2}{|c|}{ Delantero } \\
\hline & Media & Sig. & Media & Sig. & Media & Sig. & Media & Sig. \\
\hline IETET - IETERT & $.66-.74$ & .56 & $.73-.84$ & .114 & $.71-.82$ & $.036^{*}$ & $.72-.84$ & $.020 *$ \\
\hline IATACT - IATACRT & $.77-.84$ & .362 & $.75-.87$ & .093 & $.72-.86$ & $.006^{*}$ & $.67-.83$ & $.003 *$ \\
\hline IATTT - IATTRT & $.71-.78$ & .198 & $.74-.86$ & $.004 *$ & $.72-.84$ & $.013 *$ & $.69-.83$ & $.008 *$ \\
\hline
\end{tabular}

Tabla 8. Media y ANOVA: test y retest por puesto habitual de juego.

\begin{tabular}{lccccc}
\hline & Portero & Defensa & Medio & Delantero & Sig. \\
\hline IETET & .66 & .73 & .71 & .72 & .661 \\
IETERT & .74 & .84 & .82 & .84 & .199 \\
IATACT & .77 & .75 & .72 & .67 & .260 \\
IATACRT & .82 & .87 & .86 & .83 & .284 \\
IATTT & .71 & .74 & .72 & .69 & .641 \\
IATTRT & .78 & .86 & .84 & .83 & .244 \\
* $p \leq .05 /$ IETET: Índice de Ejecución-Técnica test; IATERT: Índice de Ejecución-Técnica retest; IATACT: Índice de \\
Actuación Táctica test; IATACRT: Índice de Actuación Táctica retest; IATTT: Índice de Actuación Técnico-Táctica test; \\
IATTRT: Índice de Actuación Técnico-Táctica retest.
\end{tabular}

\section{DISCUSIÓN}

La investigación tenía por objetivo analizar cómo influyen la práctica de juegos reducidos de forma interrelacionado sobre la técnica y la toma de decisiones en jugadores sub-12 de fútbol, con modificación del espacio a través de metodología alternativa.

Los resultados muestran que las variables analizadas, en cuanto a elementos de ejecucióntécnica, acciones de toma de decisiones (tácticas) y los índices de actuación mejoran tras la intervención realizada con JRs con espacio cerrado limitado por paredes. Por todo ello, se puede señalar que este tipo de intervención con JRs son una herramienta adecuada, útil y específica para la realidad del fútbol y sobre todo para los más jóvenes (Casamichana et al., 2015; Moran et al., 2019; Pérez et al., 2019; Romero-Caballero \& Campos-Vázquez, 2020), reproduciendo, en mayor medida las acciones que en espacios abiertos, lo que permite una mejora de las acciones de ejecución técnica, 
del aprendizaje situacional (táctico) y de la toma de decisiones específicas en el juego. De esta forma hay coincidencia con Owen et al. (2012) y Castejón (2015) ejercitando de forma simultánea los factores que afectan al rendimiento en la construcción del conocimiento y aprendizaje por parte del joven jugador.

Dentro del entrenamiento en el fútbol, se considera que uno de los objetivos prioritarios en las etapas iniciales es la mejora de la técnica (Katis \& Kellis, 2009; Pérez et al., 2020), en lo que se coincide completamente. Los elementos técnicos analizados mejoran de forma importante en los valores entre test-retest especialmente los pases, los controles y los disparos, además, existen diferencias significativas en el pase y el control. Estos datos sintonizan con otros estudios, que si bien no lo realizan con una muestra similar, si lo hacen con jugadores jóvenes en formación (Clemente et al. 2019a; Coutinho et al., 2020; Folgado et al., 2019; Joo et al., 2016; Pascual et al., 2017; Práxedes et al., 2018) y con los estudios de González-Víllora, García, Contreras y Gutiérrez (2010) y González-Víllora, García-López, Gutiérrez-Díaz y Pastor-Vicedo (2013) con jugadores de la misma edad, al realizar mayor número de pases y reduciendo las conducciones. La mejora en los disparos o tiros a portería coinciden con los resultados extraídos con jóvenes, con marcaje del espacio en las esquinas del campo (Coutinho et al., 2020) y con porteros en situaciones de 4x4 (Folgado et al., 2020).

A su vez, los datos anteriores, discrepan con los estudios de Pérez et al. (2019) y Sánchez et al. (2014) en la mejora de la conducción y el regate, tampoco con Pascual et al. (2017) en la mejora de la conducción lineal, ni con el estudio de Owen et al. (2004) cuando señalan que el toque libre, como es en este caso, mejora la conducción en detrimento de los controles y los pases. Tampoco con el estudio de Práxedes et al. (2018) en la mejora de los regates. De nuevo existe discrepancia con Owen at al. (2004) y Dellal et al. (2011) cuando afirman que se producen más duelos y entradas, en este caso no es así, y se puede deber al uso de la pared y al aumento de los pases y controles, de hecho, en el estudio muestran diferencias significativas la entrada, el acoso y el robo, obteniendo resultados inferiores tras la intervención.

De forma general, los resultados muestran que la media de acciones técnico-tácticas correctas, mejoran tras la intervención, coincidiendo con el estudio de Vera et al. (2008) utilizando el mismo instrumento de medida y los JRs. Existen diferencias significativas $(p<.05)$ en tres de las cuatro acciones analizadas (ATEIT-RT; ACACT-RT; ATAIRT-RT) datos que coinciden con el estudio de Aguilar, Hernández-Mendo, Martín, Reigal y Chirosa (2018). Además, se relaciona con el estudio de 
Coutinho et al. (2020) al considerar que la delimitación del espacio ayuda una menor dispersión y mejora en las decisiones realizadas por los jugadores.

La toma de decisiones basada en JRs ha sido analizada por varios autores, aunque no con el mismo instrumento de medida. Eso sí, la coincidencia es absoluta con los estudios de: Aguilar et al. (2018) donde se mejora utilizando el GPAI y la toma de decisiones global con JRs con diferencias significativas al final del estudio, como también sucede en este estudio; o con el estudio de GonzálezVillora et al. (2015) que utilizan el HERJ donde afirman que los alevines eligen mejor, o el estudio de Serra-Olivares et al. (2011) donde los jugadores deciden mejor en el tres contra tres; con otros estudios de donde se mejora en situaciones de tres contra tres utilizando para medir el GPET (Práxedes, Moreno, Sevil, García-González \& Del Villar, 2017; Práxedes, et al., 2018); también con el estudio de Pascual et al. (2017) donde el grupo de JRs mejora con respecto al grupo que no realiza este tipo de tareas y con los estudios de Farias et al. (2015), Mesquita et al. (2012) y Serra-Olivares, García-López \& Gonçalves (2019) donde se mejora la toma de decisiones con tareas jugadas de fútbol, si bien en todos estos casos lo hacen en espacio sin paredes.

Esta mejora de las acciones correctas técnico-táctico provoca que los índices de actuación totales (IATE; IATAC; IATT), mejoren más de .11 puntos, en todos los casos con diferencias significativas $(p<.000)$, por lo tanto, la toma de decisiones se mejora con la intervención realizada de forma significativa, siendo en nuestro caso superior los valores a los extraídos por Vera et al. (2008). De esta forma, el espacio cerrado permite mayor número de acciones y de toma de decisiones en consonancia con otras investigaciones (Reche-Soto et al., 2019; Romero-Caballero \& CamposVázquez, 2020).

En este sentido la mejora del pase es relacionada con la toma de decisiones en varios estudios (González-Villora et al., 2015; Pascual et al., 2017; Práxedes et al., 2017; Práxedes et al., 2018), también sucede con el dribling o regate, que se relacionan de forma directa con una mejora en la toma de decisiones de los jugadores basada en tareas de entrenamiento que impliquen a los jugadores de forma cognoscitiva (Práxedes et al., 2017), como se ha demostrado, también, en los resultados. De esta forma, al igual que Duarte, Freire, Gazimba y Araújo (2010) queda reforzada la relación directa entre la técnica y la toma de decisiones, como queda demostrado en el estudio actual, por lo tanto, se puede establecer que la toma de decisiones puede ser involucrada dentro del entrenamiento técnico (Benavides, Santos \& Díaz, 2018). 
La utilización de JRs de $3 \times 3$ con espacio cerrado con 20 sesiones de entrenamiento a través juegos reducidos como recurso metodológico dentro de la metodología innovadora debería ser tenido en cuenta como una herramienta de entrenamiento donde se relacionan los componentes cognitivos con las acciones técnico- decisionales específicas del fútbol (Benavides et al., 2018), permitiendo a los jugadores mejorar su técnica, coincidiendo con el estudio de Clemente et al. (2019a), su toma de decisiones-táctica, como se demuestra en otras investigaciones (Farias et al., 2015; González-Víllora et al., 2015; Joo et al., 2016; Moreira et al., 2020; Reche-Soto et al., 2019; Romero-Caballero \& Campos-Vázquez, 2020; Serra-Olivares et al., 2011), con especial incidencia en el juego combinativo, el aprendizaje en contextos reales y específicos (Coutinho et al., 2019; Folgado et al., 2019; Pascual et al., 2017), así como permitiendo a entrenadores y preparadores físicos desarrollar su proceso de entrenamiento-enseñanza contextualizado en el deporte, con especial incidencia en las etapas iniciales al fútbol, para preparar jugadores creativos, desarrollando sus capacidades de pensamiento y por ende de la toma de decisiones en situaciones de juego reales con contexto diferentes (Cristian et al., 2015; Coutinho et al., 2019; Folgado et al., 2019; Joo et al., 2016; Moran et al., 2019; Romero-Caballero \& Campos-Vázquez, 2020). Por lo tanto, se puede considerar que son un recurso metodológico apropiado y muy ventajoso para el entrenamiento técnico-táctico y decisional en las etapas iniciales o de formación en jugadores de fútbol con el uso de los JRs en situaciones de $3 \times 3$ con una metodología innovadora, siendo el uso de un espacio limitado por paredes como una nueva variable de entrenamiento y enseñanza.

Por último, no se realiza discusión por puestos de juego al no existir estudios que realicen este tipo de análisis. Eso sí, por puesto de juego se mejoran todos los índices y en todos los puestos de juego.

Como limitaciones, la muestra objeto de estudio es reducida y por lo tanto se necesitaría aumentar. Sería muy interesante llevar a cabo más estudios donde se analicen estos resultados en comparación con distintas categorías de fútbol y nivel competitivo, así como comparando en función del género de los deportistas y con variables de tipo condicional. Ya que la investigación ha sido reducida, por lo que los resultados no se pueden extrapolar de forma general.

\section{CONCLUSIONES}

Los resultados extraídos deben tomarse con cautela por el tipo de muestra utilizado, si bien se ha mejorado el nivel de ejecución técnica, la toma de decisiones (táctica) y la relación de ambos, a 
través de un programa de entrenamiento basado JRs con modificación del espacio y utilizando el espacio cerrado, además, la mejora se produce en un entorno de entrenamiento especifico y adaptado al nivel de los jugadores. La mejora de estos factores, decisiones y técnicos, en las edades iniciales dentro de un contexto específico es muy recomendada, para mejorar su rendimiento, así como que pueden ser de ayuda para el desarrollo en las siguientes etapas.

Por lo tanto, la utilización de JRs con limitación del espacio con paredes puede ser considerada como una variable en el diseño de tareas que debe ser contemplada en las etapas de formación de jóvenes jugadores como un elemento que presenta una buena influencia en los jugadores debido a la mejora que provoca a nivel técnico y decisional. Favoreciendo la toma de decisiones en situaciones reales de juego y de esta forma que sean capaces de dar mejor respuesta a las distintas situaciones de incertidumbre que se pueden encontrar durante los partidos, de esta forma se puede ayudar a que un equipo tome mejores decisiones de forma colectiva.

\section{REFERENCIAS}

1. Aguilar, J., Hernández-Mendo, A., Martín, I., Reigal, R.E., \& Chirosa, L.J. (2018). Efectos de unprograma de juegos reducidos sobre la toma de decisiones en chicas adolescentes. Cuadernos de Psicología del deporte, 18(1), 21-30. https://doi.org/10.4321/s1578$\underline{84232015000300008}$

2. Benavides, L.A., Santos, P.C., \& Díaz, G. (2018). La toma de decisión en el fútbol: una perspectiva desde la integración en el entrenamiento específico del deporte. Revista Ciencias de la Actividad Física UCM, 19(1), 1-10. https://doi.org/10.29035/rcaf.19.1.6

3. Casamichana, D., San Román, J., Calleja, J., \& Castellano, J. (2015). Los juegos reducidos en el entrenamiento del fútbol. Futbol de libro S.L. Editorial.

4. Castejón, F.J. (2015). La investigación en iniciación deportiva válida para el profesorado de educación física en ejercicio. Retos: Nuevas Perspectivas de Educación Física, Deporte y Recreación, 28, 263-270. https://doi.org/10.47197/retos.v0i28.35535

5. Clemente, F. M., Sarmento, H., Costa, I. T., Enes, A. R., \& Lima, R. (2019a). Variability of Technical Actions During Small-Sided Games in Young Soccer Players. Journal of Human Kinetics, 69(1), 201-212. https://doi.org/10.2478/hukin-2019-0013

6. Clemente, F.M., Rabbani, A., Kargarfard, M., Nikolaidis, P. T., Rosemann, T., \& Knechtle, B. (2019b). Session-To-Session Variations of External Load Measures of Youth Soccer Players in 
Medium-Sided Games. Int J. Environ Res Public Health, 16(19), 3612. https://doi.org/10.3390/ijerph16193612

7. Clemente, J.A.A., Suárez-Arrones, L., \& Gil, S.S. (2019c). Diferencias entre distintas orientaciones del espacio, relativizadas al perfil individual del jugador. Retos: Nuevas Tendencias en Educación Física, Deporte y Recreación, 35, 3-6. https://doi.org/10.47197/retos.v0i35.60190

8. Coutinho, D., Gonçalves, B., Santos, S., Travassos, B., Wong, D. P., \& Sampaio, J. (2019). Effects of the pitch configuration design on players' physical performance and movement behaviour during soccer small-sided games. Research in Sports Medicine, 27(3), 298-313. https://doi.org/10.1080/15438627.2018.1544133

9. Coutinho, D., Gonçalves, B., Travassos, B., Folgado, H., Figueira, B., \& Sampaio, J. (2020). Different Marks in the Pitch Constraint Youth Players' Performances During Football Smallsided Games. Research Quarterly for Exercise \& Sport, 91(1), 15-23. https://doi.org/10.1080/02701367.2019.1645938

10. Cristian, L., Riller, R., René, B., Denise, M., Milton, M., \& Alcides, S. (2015). Technical and tactical soccer players' performance in conceptual small-sided games. Motriz: Revista De Educação Física, 21(3), 312-320. https://doi.org/10.1590/S1980-65742015000300013

11. Dellal, A., Hill-Haas, S., Lago-Penas, C., \& Chamari, K. (2011). Small-sided games in soccer: amateur vs. professional players's physiological responses, physical, and technical activities. Journal of Strength \& Conditioning Research, 25(9), 2371-2381. https://doi.org/10.1519/JSC.0b013e3181fb4296

12. Duarte, R., Freire, L., Gazimba, V., \& Araújo, D. (2010). A emergência da tomada de decisão no futebol: da decisão individual para a colectiva. Nogueira, C. Psicologia do desporto: actas do VII Simpósio Nacional de Investigação em Psicologia. Minho: Universidade do Minho.

13. Farias, C. F., Ribeiro, I., \& Hastie, P. A. (2015). Game Performance and Understanding Within a Hybrid Sport Education Season. Journal of Teaching in Physical Education, 34(3), 363-383. https://doi.org/10.1123/jtpe.2013-0149

14. French, K.E.., \& Thomas, J.R. (1987). The Relation of knowledge development to children's basketball performance. Journal of Sport Psychology, 9, 15-32.

15. Folgado, H., Bravo, J., Pereira, P., \& Sampaio, J. (2019). Towards the use of multidimensional performance indicators in football small-sided games: the effects of pitch orientation. Journal of Sports Sciences, 37(9), 1064-1071. https://doi.org/10.1080/02640414.2018.1543834 
16. González-Espinosa, S., Ibáñez, S. J., \& Feu, S. (2017). Design of two basketball teaching programs in two different teaching methods. E-Balonmano.Com: Journal of Sports Science, 13(2), 131-152.

17. González-Víllora, S., García, L. M., Contreras, O. R., \& Gutiérrez, D. (2010). Estudio descriptivo sobre el desarrollo táctico y la toma de decisiones en jóvenes jugadores de fútbol (12 años). Revista Infancia y Aprendizaje, 33 (4), 489-501.

18. González-Víllora, S., García-López, L. M., Gutiérrez-Díaz, D., \& Pastor-Vicedo, J.C. (2013). Tactical awareness and decision making in youth soccer players (under-14 years). Journal of Human Sport and Exercise, 8(2), 412-426. https://doi.org/10.4100/jhse.2012.82.09

19. González-Víllora, S., García-López, L. M., \& Contreras-Jordán, O. R. (2015). Decision Making and Skill Development in Youth Football Players. Revista Internacional de Medicina y Ciencias de la Actividad Física y el Deporte, 15(59), 467-487.

20. Hill-Haas, S. V., Dawson, B., Impellizzeri, F. M., \& Coutts, A. J. (2011). Physiology of smallsided games training in football: a systematic review. Sports Medicine, 41(3), 199-220.

21. Impellizzeri, F. M., Marcora, S. M., Castagna, C., Reilly, T., Sassi, A., Iaia, F. M., \& Rampinini, E. (2006). Physiological and performance effects of generic versus specific aerobic training in soccer players. International Journal of Sports Medicine, 27(6), 483-492.

22. Joo, C. H., Hwang-Bo, K., \& Jee, H. (2016). Technical and physical activities of small-sided games in young Korean soccer players. J Strength Cond Res, 30(8), 2164-2173.

23. Katis, A., \& Kellis, E. (2009). Effects of Small Sided Games on physical conditioning and performance in young soccer players. Journal of Sports Science and Medicine, 8(3), 374-380.

24. Lacome, M., Simpson, B. M., Cholley, Y., \& Buchheit, M. (2018). Locomotor and Heart Rate Responses of Floaters During Small-Sided Games in Elite Soccer Players: Effect of Pitch Size and Inclusion of Goalkeepers. International Journal of Sports Physiology \& Performance, 13(5), 668-671. https://doi.org/10.1123/ijspp.2017-0340

25. Levi, H. R., \& Jackson, R. C. (2018). Contextual factors influencing decision making: Perceptions of professional soccer players. Psychology of Sport \& Exercise, 37, 19-25. https://doi.org/10.1016/i.psychsport.2018.04.001

26. Mesquita, I., Farias, C., \& Hastie, P. (2012). The impact of a hybrid Sport Education-Invasion Games Competence Model soccer unit on students' decision making, skill execution and overall game performance. European Physical Education Review, 18(2), 205-219.

27. Moran, J., Blagrove, R.C., Drury, B., Fernandez, J., Paxton, K., Chaabene, H., \& RámirezCampillo, R. (2019). Effects of Small-Sided Games vs. Conventional Endurance Training on 
Endurance Performance in Male Youth Soccer Players: A Meta-Analytical Comparison. Sports Med, 49(5), 731-742.

28. Moreira, A., Saldanha, M., Carling, C., Rodrigues, R. A., Schultz, A. F., Lima, M., \& ... Bradley, P. S. (2016). Temporal Changes in Technical and Physical Performances During a Small-Sided Game in Elite Youth Soccer Players. Asian Journal of Sports Medicine, 7(4), 1-8.

29. Moreira, P. E. D., Barbosa, G. F., Murta, C. D. C. F., Pérez, J. C., Bredt, S. D. G. T., Praça, G. M., \& Greco, P. J. (2020). Network analysis and tactical behaviour in soccer small-sided and conditioned games: influence of absolute and relative playing areas on different age categories. International Journal of Performance Analysis in Sport, 20(1), 64-77.

30. Nevett, M., Rovegno, I., Babiarz, M., \& MCcaughtry, N. (2001). Changes in basic tactics and motor skills in an invasion-type game after a 12-lesson unit of instruction. Journal of Teaching in Physical Education, 20(6), 352-369.

31. Owen, A. L., Wong, D. P., Paul, D., \& Dellal, A. (2012). Effects of a periodized small- sided game training intervention on physical performance in elite professional soccer. The Journal of Strength \& Conditioning Research, 26(10), 2748-2754.

32. Owen, A., Twist, C., \& Ford, P. (2004). Small-sided games: the physiological and technical effect of altering pitch size and player numbers. Insight F.A. Coaches Assoc. J, 7(2), 50-53.

33. Pascual, N., Guillén, D., \& Carbonell, J. A. (2017). Análisis comparativo de la metodología mixta y la basada en juegos reducidos en el fútbol base. Retos: Nuevas Perspectivas de Educación Física, Deporte y Recreación, 32, 199-203. https://doi.org/10.47197/retos.v0i32.56039

34. Pérez, S., Chamorro, P., Rodríguez, A., Sánchez, A., \& De Mena, J. Ma . (2020). Efecto del modelo de enseñanza sobre la técnica individual de jugadores de fútbol sub-10. SPORT TK: Revista Euroamericana de Ciencias del Deporte, 9(1), 75-84. https://doi.org/10.6018/sportk.412541

35. Pérez, S., Rodríguez, A., Sánchez, A., De Mena, J.M., Fuentes, J.M., Castaño, R., \& Martín, N. (2019). Effect of Small-Sided Games on Football Players. Revista Internacional de Medicina y Ciencias de la Actividad Física y el Deporte, 19(74), 371-386.

36. Pita, S., \& Pertega, S. (2002). Investigacion: Investigacion cuantitativa y cualitativa. Cad Aten Primaria, 9, 76-78.

37. Práxedes, A., Moreno, A., Sevil, J., García-González, D., \& Del Villar, F. (2017). The effects of a comprehensive teaching program on dribbling and passing decision-making and execution skills of young footballers. Kinesiology, 49(1), 74-83. https://doi.org/10.26582/k.49.1.6 
38. Práxedes, A., Del Villar, F., Pizarro, D., \& Moreno, A. (2018). The Impact of Nonlinear Pedagogy on Decision-Making and Execution in Youth Soccer Players According to Game Actions. Journal of Human Kinetics, 62(1), 185-198. https://doi.org/10.1515/hukin-2017-0169

39. Rampinini, E., Impellizzeri, F.M., Castagna, C., Abt, G., Chamari, K., Sassi, A., \& Marcora, S.M. (2007). Factors influencing physiological responses to small-sided soccer games. Journal of Sports Sciences, 25(6), 659-66. https://doi.org/10.1080/02640410600811858

40. Reche-Soto, P., Cardona, D., Díaz, A., Gómez-Carmona, C.D., \& Pino-Ortega, J. (2019). Tactical Demands of Small-Sided Games in Football: Influence of Tracking Technology. Revista Internacional de Medicina y Ciencias de la Actividad Física y el Deporte, 19(76), 729-744. https://doi.org/10.15366/rimcafd2019.76.011

41. Romero-Caballero, A., \& Campos-Vázquez, M. Á. (2020). Relationship between internal load indicators in a 3-a-side small-sided game in young soccer players. Retos: Nuevas Perspectivas de Educación Física, Deporte y Recreación, 37, 152-159.

42. Sánchez, J. A., Hernández-Mendo, A., Martínez, I. M., Reigal, R.E., \& Chirosa, L. J. (2018). Efectos de un programa de juegos reducidos sobre la toma de decisiones en chicas adolescentes. Cuadernos de Psicología del Deporte, 18(1), 21-30.

43. Sánchez, J., Yagüe, J.M., Fernández, R.C., \& Petisco, C. (2014). Efectos de un entrenamiento con juegos reducidos sobre la técnica y la condición física de jóvenes futbolistas. RICYDE. Revista Internacional de Ciencias del Deporte, 37(10), 221-234.

44. Santos, S., Coutinho, D., Gonçalves, B., Abade, E., Pasquarelli, B., \& Sampaio, J. (2020). Effects of manipulating ball type on youth footballers' performance during small-sided games. International Journal of Sports Science and Coaching, 15(2), 170-183.

45. Serra-Olivares, J., González-Víllora, S., \& García-López, L. M. (2011). Comparación del rendimiento de juego de jugadores de fútbol de 8-9 a-os en dos juegos modificados 3 contra 3. Cuadernos de Psicología del Deporte, 11(2), 77-91.

46. Serra-Olivares, J., García-López, L.M., Calderón, A., \& Cuevas-Campos, R. (2015). Relación del conocimiento táctico de jóvenes futbolistas con la edad, la experiencia y el nivel de pericia. Cuadernos de Psicología del Deporte, 15(3), 105-112.

47. Serra-Olivares, J., García-López, LM., \& Gonçalves, B. (2019). Effects of the players’ level and age group category on positional tactical behaviour during 7- and 8-a-side football youth games. International Journal of Performance Analysis in Sport, 19(2), 236-247. 
Pérez Muñoz et al.

48. Vera, G., Pino, J., Romero, C., \& Moreno, MI. (2007). Propuesta de valoración técnico-táctica mediante una situación de juego colectivo básico en el fútbol de iniciación. Retos: Nuevas Perspectivas de Educación Física, Deporte y Recreación, 12, 29-35.

\section{AUTHOR CONTRIBUTIONS}

All authors listed have made a substantial, direct and intellectual contribution to the work, and approved it for publication.

\section{CONFLICTS OF INTEREST}

The authors declare no conflict of interest.

\section{FUNDING}

This research received no external funding.

\section{COPYRIGHT}

(C) Copyright 2022: Publication Service of the University of Murcia, Murcia, Spain. 